UCRL-JC-119539

PREPRINT

\title{
DEVELOPMENT OF BEAM POSITION MONITORS FOR HEAVY ION RECIRCULATORS
}

\author{
F. J. Deadrick, J. J. Barnard, T. J. Fessenden, ${ }^{\dagger}$ J. W. Meridith,,$^{\ddagger}$ \\ and J. Rintamaki ${ }^{\$}$
}

\section{Lawrence Livermore National Laboratory, University of California \\ P.O. Box 5508, L-440, Livermore, California 94550, USA}

${ }^{\dagger}$ Lawrence Berkeley Laboratory,

Berkeley, California 94720, USA

RECEIVED

OCT 131995

$\ddagger E G E G$ Energy Measurements, Inc., Amador Valley Operations, OSTI 5667 Gibralter Drive, P.O. Box 8051, Pleasanton, California 94588, USA

SUniversity of Michigan, Department of Nuclear Engineering, 2355 Bonisteel Boulevard, Ann Arbor, Michigan 48109, USA

This paper was prepared for submittal to the

Proceedings of the IEEE 1995 Particle Accelerator Conference

Dallas, Texas

May 1-5, 1995

April 25, 1995

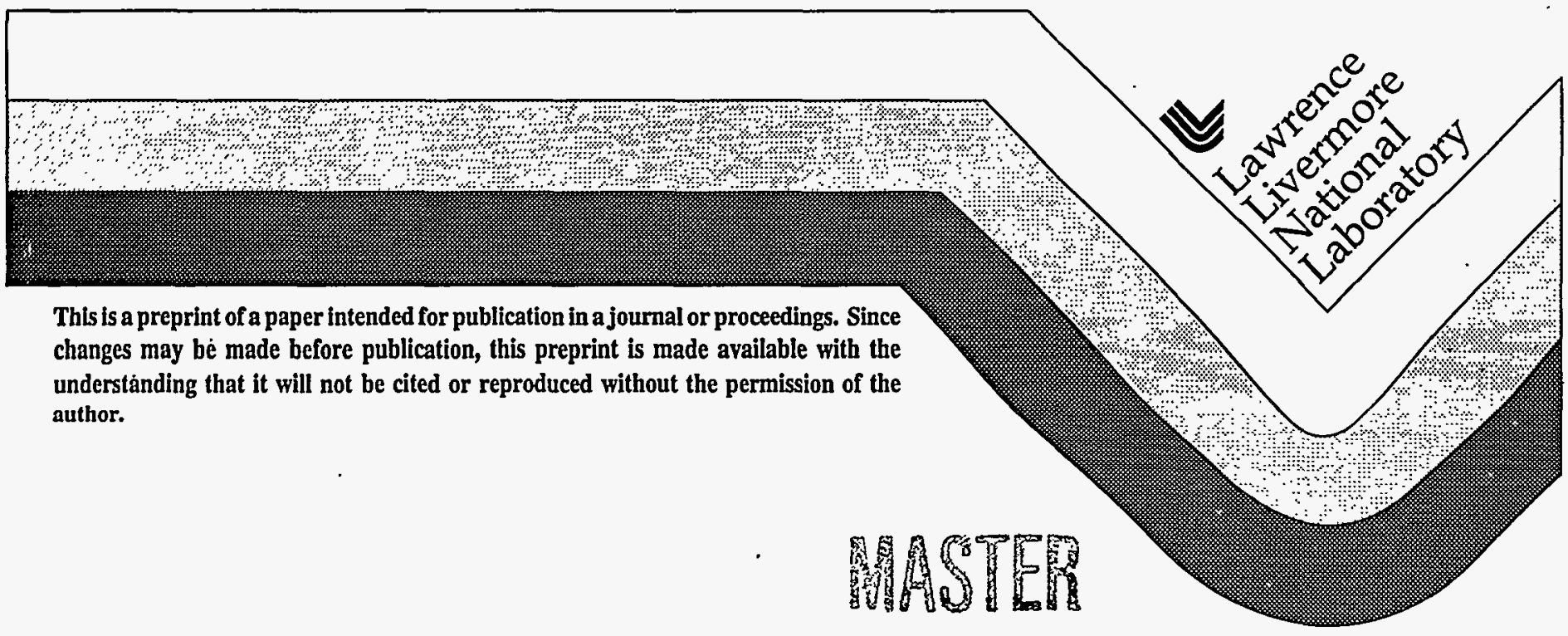

DISTFIEUTION OF THIS DOCUNAENT IS UMLIMITES

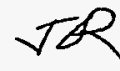




\section{DISCLAIMER}

Portions of this document may be illegible in electronic image products. Images are produced from the best available original document. 


\title{
DEVELOPMENT OF BEAM POSITION MONITORS FOR HEAVY ION RECIRCULATORS*
}

\author{
F. J. Deadrick, J. J. Barnard, Lawrence Livermore National Laboratory, Livermore, CA 94550 \\ T. J. Fessenden, LBL, J. W. Meridith, EG\&G, and J. Rintamaki, U of Mich.
}

Work is underway at the Lawrence Livermore National Laboratory to design and build a small-scale, heavy ion recirculating induction accelerator An essential part of this design work is the development of small nonintercepting diagnostics to measure beam current and position. This paper describes some of this work, with particular emphasis on the development of a small capacitive probe beam position monitor to resolve beam position to the $100 \mu \mathrm{m}$ level in a $6 \mathrm{~cm}$ diameter beam pipe. Initial measured results with an $80 \mathrm{keV}$ potassium ion beam are presented.

\section{A HEAVY ION RECIRCULATOR}

Heavy ion drivers for Inertial Confinement Fusion (ICF) offer the promise of a cost effective solution to the energy needs of the future [1]. The Lawrence Berkeley Laboratory and the Lawrence Livermore National Laboratory are cooperatively working toward the development of the technology necessary to demonstrate the feasibility of the Heavy Ion Fusion (HIF). LLNL is currently building a small-scale ( $4 \mathrm{~m}$ diameter), $4 \mu \mathrm{s}$ pulse, low-energy $(80 \mathrm{keV}$ to $320 \mathrm{keV}) \mathrm{K}^{+}$ion recirculating induction accelerator to study the technical problems associated with building a full scale recirculator [2]. Included in this work is the development of a variety of beam diagnostics which will be needed for beam monitoring and control.

\section{NON-INTERCEPTING BEAM POSITION MONITORS}

Operation in a recirculation mode requires that beam sensing diagnostics do not intercept nor áppreciably affect the beam. A wide variety of non-intercepting beam position monitors (BPM) have been devised to sense a beam's image current distribution in the beam pipe wall, the beam's image charge distribution, or the magnetic field produced by the passing beam. They all rely on the fact that the distribution of the current or charge is symmetric when the beam is on axis, however when the beam is off axis, the distribution becomes non uniform and this leads to techniques for determining beam centroid position.

Common BPM resonant tuning techniques used in RF accelerators are not always applicable to induction linacs since the beam is continuous and not RF modulated. A technique which has been successful is to use a resistive wall current monitor where the voltage drop across a resistive break is measured at several points around the circumference of the sensor, and these signals are then

* Work performed under the auspices of US DoE by LLNL under contract W-7405-ENG-48. summed and differenced to determine beam position. For this technique to be practical, however, one needs to provide electrical isolation from the beam pipe so that the current image currents flow through the resistance of the break, and azimuthal isolation during the duration of the beam pulse. This is often impractical when the beam current is low and the pulse duration is long, such as the case in our scale model accelerator.

Another technique is to sense the azimuthal magnetic fields produced by the beam current. Berners and Reginato [3] have discussed the design of a small four segment Rogowski BPM for use in the ILSE heavy ion accelerator at LBL. Again, the relatively long beam pulses and low current levels found in our recirculator make this technique difficult to implement if reasonable signal to noise ratios are expected.

Measurement of the charge distribution of the passing beam is a third candidate. Initial calculations show that this diagnostic holds the potential for providing a method of determing beam position with little impact on available space. This paper describes the development of a small non-intercepting capacitive probe beam position monitor which can be inserted inside the evacuated beampipe.

\section{CAPACITIVE PROBE PHYSICS MODEL}

Consider the design of a diagnostic as illustrated in Fig 1. Here a beam current $I_{b}$ flowing with a velocity $\beta c$ will have a charge density $\lambda$ of $I_{b} / \beta c$ coul/meter. An equal and opposite sign charge will appear in the inside surface of the beam pipe through which the beam passes. If one considers a C-Probe consisting of a segmented conductive cylinder of length $L$ inserted into the beampipe with a geometry like that shown in Fig 1, charges $\mathrm{Q}_{1}, \mathrm{Q}_{2}, \mathrm{Q}_{3}$, and $\mathrm{Q}_{4}$ are induced on the four $90^{\circ}$ sections of the conducting surface that surrounds the beam. The problem then

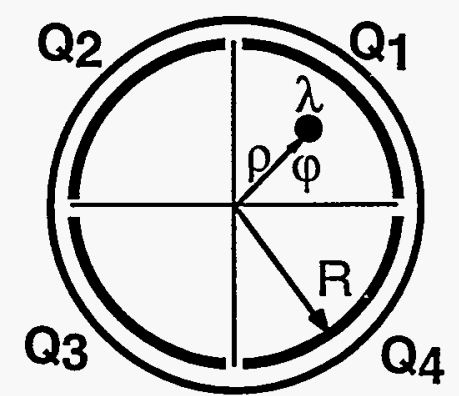

Fig. 1 Sketch of the C-probe problem and geometry

becomes one of finding an expression for the beam position and intensity in terms of the four measurable charges $Q_{1}$, $Q_{2}, Q_{3}$, and $Q_{4}$ for the general case where the beam is off 
axis. Here the charge element is a normalized distance $\rho$ off center at an angle $\varphi$ to the horizontal.

There are several methods of solving this field problem. The most customary way is by the method of images wherein an image line-charge of value $-\lambda$ at an angle $\varphi$ and position $R / \rho$ from the origin is assumed. It can be shown that the circle of radius $R$ is an equipotential in the field of the two line charges. The field component normal to the conduction surface gives the distribution of charge around its circumference. From this distribution the total charge on each $90^{\circ}$ section can be calculated and the voltage on each section determined. Note we have assumed that the gaps between plates do not significantly change the distribution of charges. One finds the charge on the circumference to be given by

$$
\sigma(\vartheta)=\frac{\lambda}{2 \pi R}\left[\frac{1-\rho^{2}}{1+\rho^{2}-2 \rho \cos (\vartheta-\varphi)}\right]
$$

were $\sigma(\vartheta)$ is the surface charge distribution on the conducting surfaces. Upon integrating this expression over the conducting surface at $R$ (easier using complex variable techniques) one obtains the sum of the four charges $\mathrm{Q}_{1}$ $+Q_{2}+Q_{3}+Q_{4}=\lambda L$ as expected. Here $L$ is the axial length of the $\mathrm{C}$-probe. The total charge on the first BPM sector is then given by

$$
\mathrm{Q}_{1} / \mathrm{L}=\frac{\lambda}{2 \pi} \int_{0}^{\pi / 2} \frac{1-\rho^{2}}{1+\rho^{2}-2 \rho \cos (\vartheta-\varphi)} d \vartheta
$$

Similar expressions exist for the other three sectors.

Consider the following definitions:

$$
X \equiv \frac{\left(Q_{1}+Q_{4}-Q_{2}-Q_{3}\right)}{\left(Q_{1}+Q_{2}+Q_{3}+Q_{4}\right)}
$$

and

$$
Y \equiv \frac{\left(Q_{1}+Q_{2}-Q_{3}-Q_{4}\right)}{\left(Q_{1}+Q_{2}+Q_{3}+Q_{4}\right)} \text {. }
$$

Using the expression for the charge around the circumference given in Eq. (1), and integrating from 0 to $\pi / 2$ as in Eq. (2), one finds an expression for the variable $X$ to be given by

$$
X=\frac{1}{2 \pi}\left\{\begin{array}{l}
4 \tan ^{-1}\left[\frac{1+\rho}{1-\rho} \tan (\pi / 4+\varphi / 2)\right]+ \\
4 \tan ^{-1}\left[\frac{1+\rho}{1-\rho} \tan (\pi / 4-\varphi / 2)\right]-2 \pi
\end{array}\right\}
$$

In terms of the Cartesian coordinates, one finds $X=$

$$
\frac{1}{2 \pi}\left\{\begin{array}{l}
4 \tan ^{-1}\left[\frac{1+\sqrt{x^{2}+y^{2}}}{1-\sqrt{x^{2}+y^{2}}} \tan \left(\pi / 4+\frac{\tan ^{-1}(y / x)}{2}\right)\right] . \\
+4 \tan ^{-1}\left[\frac{1+\sqrt{x^{2}+y^{2}}}{1-\sqrt{x^{2}+y^{2}}} \tan \left(\pi / 4-\frac{\tan ^{-1}(y / x)}{2}\right)\right]-2 \pi
\end{array}\right\}
$$

Here $x$ and $y$ are the spatial coordinates divided by the pipe radius $R$. A similar expression can be found for $Y$.

One can show that $X$ and $Y$ are zero at the origin. For small values of $x$ and $y$, or for beams near the center axis,

$$
\begin{aligned}
& X \approx 4 / \pi x ; \quad Y \approx 4 / \pi y \quad(x, y<1) \quad \text { or } \\
& X \approx \pi / 4 X ; y \approx \pi / 4 Y .
\end{aligned}
$$

Within the circle $\sqrt{\mathrm{x}^{2}+\mathrm{y}^{2}}=0.5$, the approximate expressions given above are accurate to better than $7 \%$. Within $\sqrt{x^{2}+y^{2}}=0.25$ the error becomes less than $2 \%$. For most situations this should be adequate for determining the beam position within the diagnostic. Needless-to-say, one must multiply the variables $\mathrm{x}$ and $\mathrm{y}$ by the pipe radius $R$ to obtain the actual offset in centimeters or inches.

Fig 2. shows a contour plot of both functions $X$ and $Y$ for values from -0.9 to 0.9 in steps of 0.1 . Thus for a particular experimental situation, if the values of $X$ and $Y$ are measured, the position of the beam within the pipe and be determined.

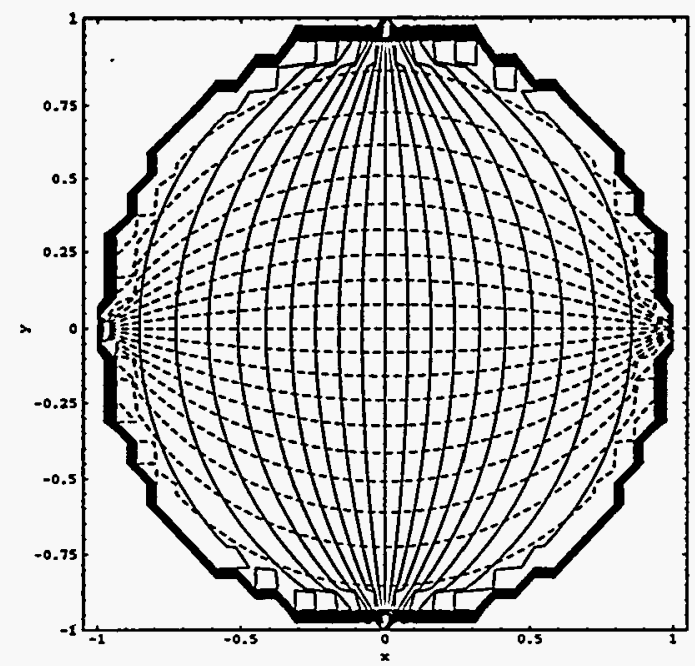

Fig. 2. Contour plots of $X$ and $Y$. The solid lines are contours of constant $X$ in increments of 0.1 . The dotted lines are corresponding contours of constant $Y$.

\section{ELECTRICAL CONSIDERATIONS}

A circuit of the type shown in Fig. 3 will produce signal voltages proportional to $\mathrm{X}$ and $\mathrm{Y}$. Here the charge on the four sectors is converted to a voltage signal by the capacitance of the sensor including any external capacitance, $\mathrm{C}_{\text {ext }}$. Since the sensor voltage output is inversely proportional to the total system capacitance, it is desirable to locate the buffer amplifiers as close to the sensor as practical.

As an example, the total charge induced on the probe sectors is simply the beam line-charge-density times the axial length of the sector, $L$. For a sector length of $10 \mathrm{~cm}$, and a $2 \mathrm{~mA}$ potassium beam at $80 \mathrm{kV}(\beta=0.002)$, we find that approximately $0.33 \mathrm{nC}$ total or $0.083 \mathrm{nC}$ per sector will appear on the plates. If the system capacitance is $1 \mathrm{nF}$, then the voltage on each plate will be near 80 millivolts. Note 
that it is especially important to keep the effective capacitance of each of the four circuits extremely well balanced if accurate position information is to be obtained.

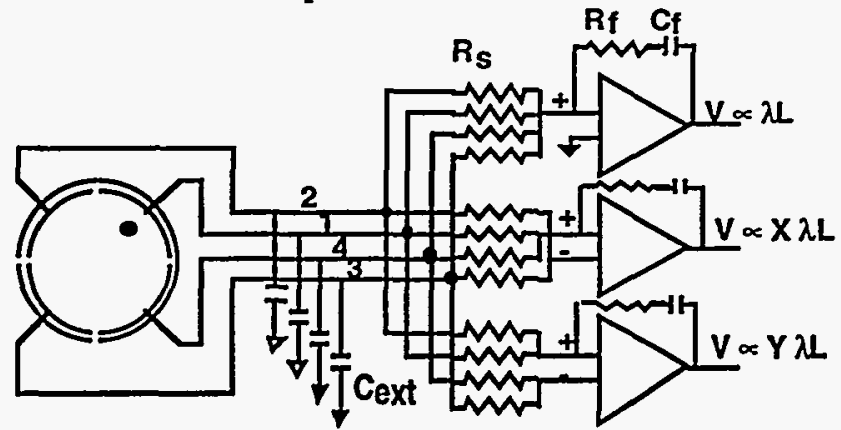

Fig. 3. Monitor circuit to give an electrical output proportional to the total charge, and proportional to the beam displacements in $\mathrm{X}$ and $\mathrm{Y}$.

\section{TEST RESULTS \& CONCLUSIONS}

A prototype C-Probe has been built and tested with an ion beam [4]. The sensor element is made on a Macor glass-ceramic cylinder $11.2 \mathrm{~cm}$ long by $5.7 \mathrm{~cm}$ in diameter. Copper sensor plates are deposited on the inside surface of the cylinder using an electroless plating process. The cylinder fits inside a beampipe which is surrounded by a permanent magnet quadrupole. The intrinsic capacitance of each of the four segments has been measured at $10 \mathrm{pF}$. However, external cabling used for monitoring and recording the probe signals brought the total capacitance of the circuit up to approximately $2 \mathrm{nF}$. Fig 4 shows the measured waveforms on each of the four plates due to a $4 \mu \mathrm{s}$ wide, $2 \mathrm{ma} \mathrm{K}{ }^{+}$ion beam pulse.

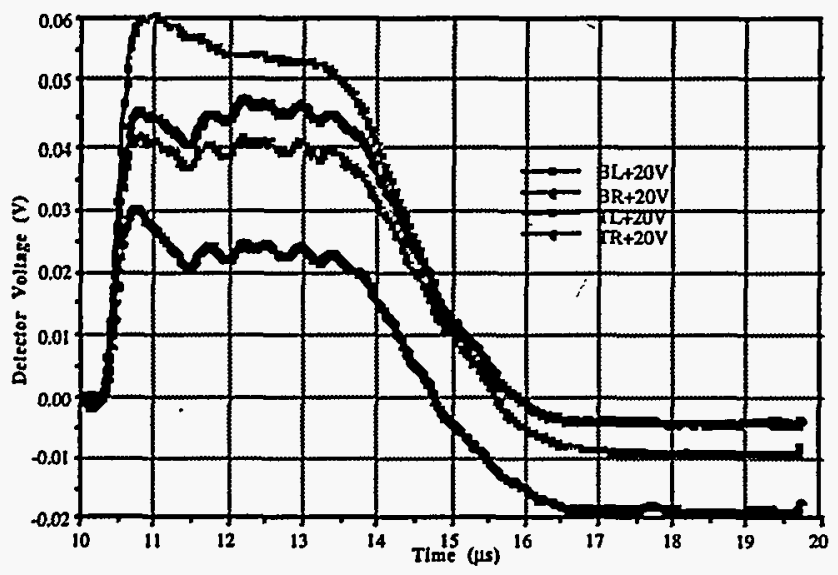

Fig 4. Measured C-Probe plate voltages for a 2.1 ma beam.

Figure 5 shows the waveforms obtained if one numerically adds and subtracts the individual plate voltages as detailed in Fig. 3, where $X$ and $Y$ are proportional to the distance that the ion beam is off axis. By using the average values of the voltages during the beam pulse as shown, one can calculate that the beam is off axis by $-3.3 \mathrm{~mm}$ in the $x$ direction, and $+4 \mathrm{~mm}$ in the y direction inside a $5.7 \mathrm{~cm}$ diameter beampipe. These offset predictions are quite reasonable considering that a final mechanical alignment of the beamline had not been performed prior to these measurements. Beam profile diagnostic instrumentation is currently being installed to determine absolute beam centroid position. An insitu calibration will be needed to verify the calculated sensitivity factors and balance the analog readout electronics.

Further work certainly remains to turn this diagnostic into a reliable BPM. One of the concerns in using a charge sensitive diagnostic of this type is the affect that stray electrons hitting the sensor plates will have on the plate charge. More importantly the affect of ions hitting the plates is also a factor. We have found that secondary electrons at a ratio of approximately 8 electrons per ion are produced. For this reason a positive bias voltage on the electrodes should be used to re-collect any secondary electrons.

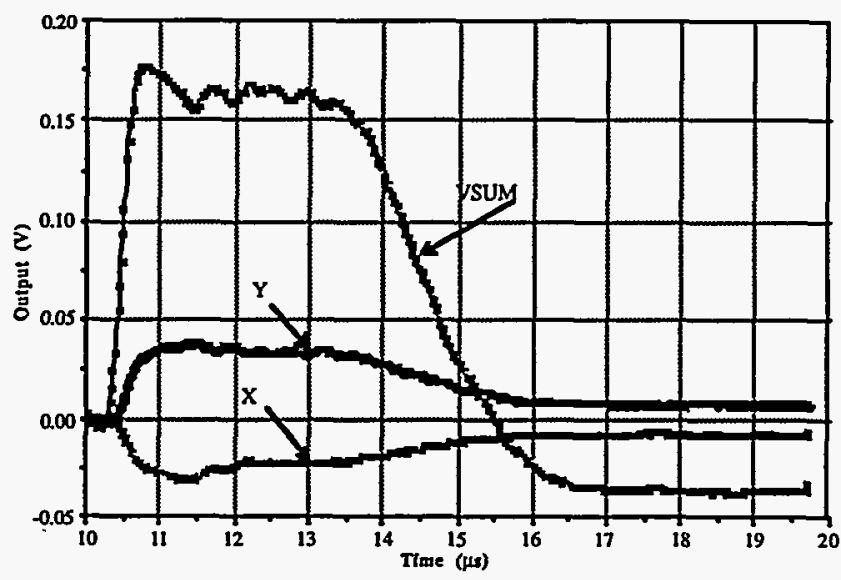

Fig. 5. Processed X \& Y offset signals from C-Probe.

Finally, care is needed in the design of the instrumentation electronics. The monitoring circuitry should measure the charge on the capacitors, and not significantly deplete this charge during the beam pulse time. The charge waveforms should return to zero after the beam pulse has passed the sensor.

We find these initial results to be encouraging, and plan to continue the development of this simple diagnostic.

\section{REFERENCES}

[1] J. J. Barnard, et. al., "Study of Recirculating Induction Accelerators as Drivers for Heavy Ion Fusion", Lawrence Livermore National Lab, UCRL-LR-108095, September 21, 1991.

[2] A. Friedman, et. al., "Progress Toward a Prototype Recirculating Induction Accelerator for Heavy Ion Fusion", This Conference,

[3] D. Berners and L Reginato, "Beam Position and Total Current Monitor for Heavy Ion Fusion Beams", Accelerator Instrumentation Fourth Annual Workshop, AIP Conference Proceedings No. 281, Berkeley, CA

[4] S. Eylon, E. Henestroza and F. Deadrick, "K+Diode for the LLNL Heavy Ion Recirculator Accelerator Experiment", This Conference. 